\title{
Generalized Golub-Kahan bidiagonalization and stopping criteria
}

\author{
Mario Arioli
}

STFC Rutherford Appleton Laboratory collaboration with Dominique Orban

GERAD and École Polytechnique de Montréal

Granlibakken 13 June 2011 


\section{Overview of talk}

- Augmented Systems

- Symmetric Quasi Positive Definite matrices 


\section{Overview of talk}

- Augmented Systems

- Generalized singular values and minimization problem

- G-K bidiagonalization

- Stopping criteria

- Numerical examples

- Symmetric Quasi Positive Definite matrices

- Work in progress in collaboration with Dominique Orban (GERAD and École Polytechnique de Montréal) 


\section{Linear operators}

Let $\mathbf{M} \in \mathrm{R}^{m \times m}$ and $\mathbf{N} \in \mathrm{R}^{n \times n}$ be symmetric positive definite matrices, and let $\mathbf{A} \in \mathbf{R}^{m \times n}$ be a full rank matrix.

$$
\begin{gathered}
\mathcal{M}=\left\{\mathbf{v} \in \mathbf{R}^{m} ;\|\mathbf{u}\|_{\mathbf{M}}^{2}=\mathbf{v}^{T} \mathbf{M} \mathbf{v}\right\}, \mathcal{N}=\left\{\mathbf{q} \in \mathbf{R}^{n} ;\|\mathbf{q}\|_{\mathbf{N}}^{2}=\mathbf{q}^{T} \mathbf{N} \mathbf{q}\right\} \\
\mathcal{M}^{\prime}=\left\{\mathbf{w} \in \mathbf{R}^{m} ;\|\mathbf{w}\|_{\mathbf{M}^{-1}}^{2}=\mathbf{w}^{T} \mathbf{M}^{-1} \mathbf{w}\right\} \\
\mathcal{N}^{\prime}=\left\{\mathbf{y} \in \mathbf{R}^{n} ;\|\mathbf{y}\|_{\mathbf{N}^{-1}}^{2}=\mathbf{y}^{T} \mathbf{N}^{-1} \mathbf{y}\right\}
\end{gathered}
$$




\section{Linear operators}

Let $\mathbf{M} \in \mathrm{R}^{m \times m}$ and $\mathbf{N} \in \mathrm{R}^{n \times n}$ be symmetric positive definite matrices, and let $\mathbf{A} \in \mathbf{R}^{m \times n}$ be a full rank matrix.

$$
\begin{gathered}
\mathcal{M}=\left\{\mathbf{v} \in \mathbf{R}^{m} ;\|\mathbf{u}\|_{\mathbf{M}}^{2}=\mathbf{v}^{T} \mathbf{M} \mathbf{v}\right\}, \mathcal{N}=\left\{\mathbf{q} \in \mathbf{R}^{n} ;\|\mathbf{q}\|_{\mathbf{N}}^{2}=\mathbf{q}^{T} \mathbf{N} \mathbf{q}\right\} \\
\mathcal{M}^{\prime}=\left\{\mathbf{w} \in \mathbf{R}^{m} ;\|\mathbf{w}\|_{\mathbf{M}^{-1}}^{2}=\mathbf{w}^{T} \mathbf{M}^{-1} \mathbf{w}\right\} \\
\mathcal{N}^{\prime}=\left\{\mathbf{y} \in \mathbf{R}^{n} ;\|\mathbf{y}\|_{\mathbf{N}^{-1}}^{2}=\mathbf{y}^{T} \mathbf{N}^{-1} \mathbf{y}\right\} \\
\langle\mathbf{v}, \mathbf{A} \mathbf{q}\rangle_{\mathcal{M}, \mathcal{M}^{\prime}}=\mathbf{v}^{T} \mathbf{A} \mathbf{q}, \quad \mathbf{A q} \in \mathcal{L}(\mathcal{M}) \forall \mathbf{q} \in \mathcal{N}
\end{gathered}
$$




\section{Linear operators}

Let $\mathbf{M} \in \mathrm{R}^{m \times m}$ and $\mathbf{N} \in \mathrm{R}^{n \times n}$ be symmetric positive definite matrices, and let $\mathbf{A} \in \mathbf{R}^{m \times n}$ be a full rank matrix.

$$
\begin{gathered}
\mathcal{M}=\left\{\mathbf{v} \in \mathbf{R}^{m} ;\|\mathbf{u}\|_{\mathbf{M}}^{2}=\mathbf{v}^{T} \mathbf{M} \mathbf{v}\right\}, \mathcal{N}=\left\{\mathbf{q} \in \mathbf{R}^{n} ;\|\mathbf{q}\|_{\mathbf{N}}^{2}=\mathbf{q}^{T} \mathbf{N} \mathbf{q}\right\} \\
\mathcal{M}^{\prime}=\left\{\mathbf{w} \in \mathbf{R}^{m} ;\|\mathbf{w}\|_{\mathbf{M}^{-1}}^{2}=\mathbf{w}^{T} \mathbf{M}^{-1} \mathbf{w}\right\} \\
\mathcal{N}^{\prime}=\left\{\mathbf{y} \in \mathbf{R}^{n} ;\|\mathbf{y}\|_{\mathbf{N}^{-1}}^{2}=\mathbf{y}^{T} \mathbf{N}^{-1} \mathbf{y}\right\} \\
\langle\mathbf{v}, \mathbf{A} \mathbf{q}\rangle_{\mathcal{M}, \mathcal{M}^{\prime}}=\mathbf{v}^{T} \mathbf{A} \mathbf{q}, \quad \mathbf{A q} \in \mathcal{L}(\mathcal{M}) \forall \mathbf{q} \in \mathcal{N} .
\end{gathered}
$$

The adjoint operator $\mathbf{A}^{\star}$ of $\mathbf{A}$ can be defined as

$$
\left\langle\mathbf{A}^{\star} \mathbf{g}, \mathbf{f}\right\rangle_{\mathcal{N}^{\prime}, \mathcal{N}}=\mathbf{f}^{T} \mathbf{A}^{T} \mathbf{g}, \quad \mathbf{A}^{T} \mathbf{g} \in \mathcal{L}(\mathcal{N}) \forall \mathbf{g} \in \mathcal{M} .
$$




\section{Generalized SVD}

Given $\mathbf{q} \in \mathcal{M}$ and $\mathbf{v} \in \mathcal{N}$, the critical points for the functional

$$
\frac{\mathbf{v}^{T} \mathbf{A q}}{\|\mathbf{q}\|_{\mathrm{N}}\|\mathbf{v}\|_{\mathrm{M}}}
$$

are the "generalized singular values and singular vectors" of $\mathbf{A}$. 


\section{Generalized SVD}

Given $\mathbf{q} \in \mathcal{M}$ and $\mathbf{v} \in \mathcal{N}$, the critical points for the functional

$$
\frac{\mathbf{v}^{T} \mathbf{A q}}{\|\mathbf{q}\|_{\mathrm{N}}\|\mathbf{v}\|_{\mathrm{M}}}
$$

are the "generalized singular values and singular vectors" of $\mathbf{A}$.

The saddle-point conditions are

$$
\begin{gathered}
\begin{cases}\mathbf{A q}_{i}=\sigma_{i} \mathbf{M} \mathbf{v}_{i} & \mathbf{v}_{i}^{T} \mathbf{M} \mathbf{v}_{j}=\delta_{i j} \\
\mathbf{A}^{T} \mathbf{v}_{i}=\sigma_{i} \mathbf{N} \mathbf{q}_{i} \quad & \mathbf{q}_{i}^{T} \mathbf{N} \mathbf{q}_{j}=\delta_{i j}\end{cases} \\
\sigma_{1} \geq \sigma_{2} \geq \cdots \geq \sigma_{n}>0
\end{gathered}
$$




\section{Generalized SVD}

Given $\mathbf{q} \in \mathcal{M}$ and $\mathbf{v} \in \mathcal{N}$, the critical points for the functional

$$
\frac{\mathbf{v}^{T} \mathbf{A q}}{\|\mathbf{q}\|_{\mathrm{N}}\|\mathbf{v}\|_{\mathrm{M}}}
$$

are the "generalized singular values and singular vectors" of $\mathbf{A}$.

The saddle-point conditions are

$$
\begin{aligned}
& \left\{\mathbf{A q}_{i}=\sigma_{i} \mathbf{M v}_{i} \quad \mathbf{v}_{i}^{T} \mathbf{M v}_{j}=\delta_{i j}\right. \\
& \mathbf{A}^{T} \mathbf{v}_{i}=\sigma_{i} \mathbf{N} \mathbf{q}_{i} \quad \mathbf{q}_{i}^{T} \mathbf{N q}_{j}=\delta_{i j} \\
& \sigma_{1} \geq \sigma_{2} \geq \cdots \geq \sigma_{n}>0
\end{aligned}
$$

The generalized singular values are the standard singular values of $\tilde{\mathbf{A}}=\mathbf{M}^{-1 / 2} \mathbf{A} \mathbf{N}^{-1 / 2}$. The generalized singular vectors $\mathbf{q}_{i}$ and $\mathbf{v}_{i}$, $i=1, \ldots, n$ are the transformation by $\mathbf{M}^{-1 / 2}$ and $\mathbf{N}^{-1 / 2}$ respectively of the left and right standard singular vector of $\tilde{\mathbf{A}}$. 


\section{Quadratic programming}

The general problem

$$
\min _{\mathbf{A}^{T} \mathbf{w}=\mathbf{r}} \frac{1}{2} \mathbf{w}^{T} \mathbf{W} \mathbf{w}-\mathbf{g}^{T} \mathbf{w}
$$

where the matrix $\mathbf{W}$ is positive semidefinite and $\operatorname{ker}(\mathbf{W}) \cap \operatorname{ker}\left(\mathbf{A}^{T}\right)=0$ can be reformulated by choosing

$$
\begin{aligned}
& \mathbf{M}=\mathbf{W}+\nu \mathbf{A} \mathbf{N}^{-1} \mathbf{A}^{T} \\
& \mathbf{u}=\mathbf{w}-\mathbf{M}^{-1} \mathbf{g} \\
& \mathbf{b}=\mathbf{r}-\mathbf{A}^{T} \mathbf{M}^{-1} \mathbf{g}
\end{aligned}
$$

as a projection problem

$$
\min _{\mathbf{A}^{T} \mathbf{u}=\mathbf{b}}\|\mathbf{u}\|_{\mathbf{M}}^{2}
$$

If $\mathbf{W}$ is non singular then we can choose $\nu=0$. 


\section{Augmented system}

The augmented system that gives the optimality conditions for the projection problem:

$$
\left[\begin{array}{cc}
\mathbf{M} & \mathbf{A} \\
\mathbf{A}^{T} & 0
\end{array}\right]\left[\begin{array}{l}
\mathbf{u} \\
\mathbf{p}
\end{array}\right]=\left[\begin{array}{l}
0 \\
\mathbf{b}
\end{array}\right] .
$$




\section{Generalized Golub-Kahan bidiagonalization}

In Golub Kahan (1965), Paige Saunders (1982), several algorithms for the bidiagonalization of a $m \times n$ matrix are presented. All of them can be theoretically applied to $\tilde{\mathbf{A}}$ and their generalization to A is straightforward as shown by Bembow (1999). Here, we want specifically to analyse one of the variants known as the "Craig"-variant (see Paige Saunders (1982), Saunders $(1995,1997))$. 


\section{Generalized Golub-Kahan bidiagonalization}

$$
\left\{\begin{array}{lll}
\mathbf{A} \tilde{\mathbf{Q}}=\mathbf{M} \tilde{\mathbf{V}}\left[\begin{array}{c}
\tilde{\mathbf{B}} \\
0
\end{array}\right] & \tilde{\mathbf{V}}^{T} \mathbf{M} \tilde{\mathbf{V}}=\mathbf{I}_{m} \\
\mathbf{A}^{T} \tilde{\mathbf{V}}=\mathbf{N} \tilde{\mathbf{Q}}\left[\tilde{\mathbf{B}}^{T} ; 0\right] & \tilde{\mathbf{Q}}^{T} \mathbf{N} \tilde{\mathbf{Q}}=\mathbf{I}_{n}
\end{array}\right.
$$

where

$$
\tilde{\mathbf{B}}=\left[\begin{array}{ccccc}
\tilde{\alpha}_{1} & 0 & 0 & \cdots & 0 \\
\tilde{\beta}_{2} & \tilde{\alpha}_{2} & 0 & \ddots & 0 \\
\vdots & \ddots & \ddots & \ddots & \ddots \\
0 & \cdots & \tilde{\beta}_{n-1} & \tilde{\alpha}_{n-1} & 0 \\
0 & \cdots & 0 & \tilde{\beta}_{n} & \tilde{\alpha}_{n} \\
0 & \cdots & 0 & 0 & \tilde{\beta}_{n+1}
\end{array}\right]
$$




\section{Generalized Golub-Kahan bidiagonalization}

$$
\begin{cases}\mathbf{A} \mathbf{Q}=\mathbf{M V}\left[\begin{array}{c}
\mathbf{B} \\
0
\end{array}\right] & \mathbf{V}^{T} \mathbf{M V}=\mathbf{I}_{m} \\
\mathbf{A}^{T} \mathbf{V}=\mathbf{N Q}\left[\mathbf{B}^{T} ; 0\right] & \mathbf{Q}^{T} \mathbf{N} \mathbf{Q}=\mathbf{I}_{n}\end{cases}
$$

where

$$
\mathbf{B}=\left[\begin{array}{ccccc}
\alpha_{1} & \beta_{1} & 0 & \cdots & 0 \\
0 & \alpha_{2} & \beta_{2} & \ddots & 0 \\
\vdots & \ddots & \ddots & \ddots & \ddots \\
0 & \cdots & 0 & \alpha_{n-1} & \beta_{n-1} \\
0 & \cdots & 0 & 0 & \alpha_{n}
\end{array}\right]
$$




\section{Algorithm}

The augmented system that gives the optimality conditions for $\min _{\mathbf{A}^{T} \mathbf{u}=\mathbf{b}}\|\mathbf{u}\|_{\mathbf{M}}^{2}$

$$
\left[\begin{array}{cc}
\mathbf{M} & \mathbf{A} \\
\mathbf{A}^{T} & 0
\end{array}\right]\left[\begin{array}{l}
\mathbf{u} \\
\mathbf{p}
\end{array}\right]=\left[\begin{array}{l}
0 \\
\mathbf{b}
\end{array}\right]
$$

can be transformed by the change of variables

$$
\left\{\begin{array}{l}
\mathbf{u}=\mathbf{V} \mathbf{z} \\
\mathbf{p}=\mathbf{Q} \mathbf{y}
\end{array}\right.
$$




\section{Algorithm}

$\left[\begin{array}{ccc}\mathbf{I}_{n} & 0 & \mathbf{B} \\ 0 & \mathbf{I}_{m-n} & 0 \\ \mathbf{B}^{T} & 0 & 0\end{array}\right]\left[\begin{array}{c}\mathbf{z}_{1} \\ \mathbf{z}_{2} \\ \mathbf{y}\end{array}\right]=\left[\begin{array}{c}0 \\ 0 \\ \mathbf{Q}^{T} \mathbf{b}\end{array}\right]$ 


\section{Algorithm}

$$
\left[\begin{array}{ll}
\mathbf{I}_{n} & \mathbf{B} \\
\mathbf{B}^{T} & 0
\end{array}\right]\left[\begin{array}{c}
\mathbf{z}_{1} \\
\mathbf{y}
\end{array}\right]=\left[\begin{array}{c}
0 \\
\mathbf{Q}^{T} \mathbf{b}
\end{array}\right] .
$$




\section{Algorithm}

$$
\begin{gathered}
{\left[\begin{array}{cc}
\mathbf{I}_{n} & \mathbf{B} \\
\mathbf{B}^{T} & 0
\end{array}\right]\left[\begin{array}{c}
\mathbf{z}_{1} \\
\mathbf{y}
\end{array}\right]=\left[\begin{array}{c}
0 \\
\mathbf{Q}^{T} \mathbf{b}
\end{array}\right] .} \\
\mathbf{Q}^{T} \mathbf{b}=\mathbf{e}_{1}\|\mathbf{b}\|_{\mathbf{N}}
\end{gathered}
$$

the value of $\mathbf{z}_{1}$ will correspond to the first column of the inverse of B multiplied by $\|\mathbf{b}\|_{\mathbf{N}}$. 


\section{Algorithm}

Thus, we can compute the first column of $\mathbf{B}$ and of $\mathbf{V}$ : $\alpha_{1} \mathbf{M v}_{1}=\mathbf{A} \mathbf{q}_{1}$, such as

$$
\begin{aligned}
& \mathbf{w}=\mathbf{M}^{-1} \mathbf{A q}_{1} \\
& \alpha_{1}=\mathbf{w}^{T} \mathbf{M} \mathbf{w}=\mathbf{w A q}_{1} \\
& \mathbf{v}_{1}=\mathbf{w} / \sqrt{\alpha_{1}} .
\end{aligned}
$$




\section{Algorithm}

Thus, we can compute the first column of $\mathbf{B}$ and of $\mathbf{V}$ :

$\alpha_{1} \mathbf{M} \mathbf{v}_{1}=\mathbf{A} \mathbf{q}_{1}$, such as

$$
\begin{aligned}
& \mathbf{w}=\mathbf{M}^{-1} \mathbf{A} \mathbf{q}_{1} \\
& \alpha_{1}=\mathbf{w}^{T} \mathbf{M} \mathbf{w}=\mathbf{w} \mathbf{A} \mathbf{q}_{1} \\
& \mathbf{v}_{1}=\mathbf{w} / \sqrt{\alpha_{1}} .
\end{aligned}
$$

Finally, knowing $\mathbf{q}_{1}$ and $\mathbf{v}_{1}$ we can start the recursive relations

$$
\begin{aligned}
& \mathbf{g}_{i+1}=\mathbf{N}^{-1}\left(\mathbf{A}^{T} \mathbf{v}_{i}-\alpha_{i} \mathbf{N} \mathbf{q}_{i}\right) \\
& \beta_{i+1}=\mathbf{g}^{T} \mathbf{N g} \\
& \mathbf{q}_{i+1}=\mathbf{g} \sqrt{\beta_{i+1}} \\
& \mathbf{w}=\mathbf{M}^{-1}\left(\mathbf{A} \mathbf{q}_{i+1}-\beta_{i+1} \mathbf{M} \mathbf{v}_{i}\right) \\
& \alpha_{i+1}=\mathbf{w}^{T} \mathbf{M} \mathbf{w} \\
& \mathbf{v}_{i+1}=\mathbf{w} / \sqrt{\alpha_{i+1}} .
\end{aligned}
$$




\section{$\mathbf{u}$}

Thus, the value of $\mathbf{u}$ can be approximated when we have computed the first $k$ columns of $\mathbf{U}$ by

$$
\mathbf{u}^{(k)}=\mathbf{V}_{k} \mathbf{z}_{k}=\sum_{j=1}^{k} \zeta_{j} \mathbf{v}_{j}
$$


$\mathbf{U}$

Thus, the value of $\mathbf{u}$ can be approximated when we have computed the first $k$ columns of $\mathbf{U}$ by

$$
\mathbf{u}^{(k)}=\mathbf{V}_{k} \mathbf{z}_{k}=\sum_{j=1}^{k} \zeta_{j} \mathbf{v}_{j}
$$

The entries $\zeta_{j}$ of $\mathbf{z}_{k}$ can be easily computed recursively starting with

$$
\zeta_{1}=-\frac{\|\mathbf{b}\|_{\mathbf{N}}}{\alpha_{1}}
$$

as

$$
\zeta_{i+1}=-\frac{\beta_{i}}{\alpha_{i+1}} \zeta_{i} \quad i=1, \ldots, n
$$


$p$

Approximating $\mathbf{p}=\mathbf{Q} \mathbf{y}$ by $\mathbf{p}^{(k)}=\mathbf{Q}_{k} \mathbf{y}_{k}=\sum_{j=1}^{k} \psi_{j} \mathbf{q}_{j}$, we have that

$$
\mathbf{y}_{k}=-\mathbf{B}_{k}^{-1} \mathbf{z}_{k} \text {. }
$$


$p$

Approximating $\mathbf{p}=\mathbf{Q} \mathbf{y}$ by $\mathbf{p}^{(k)}=\mathbf{Q}_{k} \mathbf{y}_{k}=\sum_{j=1}^{k} \psi_{j} \mathbf{q}_{j}$, we have that

$$
\mathbf{y}_{k}=-\mathbf{B}_{k}^{-1} \mathbf{z}_{k} .
$$

Following an observation made by Paige and Saunders, we can easily transform the previous relation into a recursive one where only one extra vector is required. 
Approximating $\mathbf{p}=\mathbf{Q} \mathbf{y}$ by $\mathbf{p}^{(k)}=\mathbf{Q}_{k} \mathbf{y}_{k}=\sum_{j=1}^{k} \psi_{j} \mathbf{q}_{j}$, we have that

$$
\mathbf{y}_{k}=-\mathbf{B}_{k}^{-1} \mathbf{z}_{k} \text {. }
$$

$$
\begin{gathered}
\text { From } \mathbf{p}^{(k)}=-\mathbf{Q}_{k} \mathbf{B}_{k}^{-1} \mathbf{z}_{k}=-\left(\mathbf{B}_{k}^{-T} \mathbf{Q}_{k}^{T}\right)^{T} \mathbf{z}_{k} \text { and } \mathbf{D}_{k}=\mathbf{B}_{k}^{-T} \mathbf{Q}_{k}^{T} \\
\mathbf{d}_{i}=\frac{\mathbf{q}_{i}-\beta_{i} \mathbf{d}_{i-1}}{\alpha_{i}} \quad i=1, \ldots, n \quad\left(\mathbf{d}_{0}=0\right)
\end{gathered}
$$

where $\mathbf{d}_{j}$ are the columns of $\mathbf{D}$.

Starting with $\mathbf{p}^{(1)}=-\zeta_{1} \mathbf{d}_{1}$ and $\mathbf{u}^{(1)}=\zeta_{1} \mathbf{v}_{1}$

$$
\left.\begin{array}{l}
\mathbf{u}^{(i+1)}=\mathbf{u}^{(i)}+\zeta_{i+1} \mathbf{v}_{i+1} \\
\mathbf{p}^{(i+1)}=\mathbf{p}^{(i)}-\zeta_{i+1} \mathbf{d}_{i+1}
\end{array}\right\} \quad i=1, \ldots, n
$$




\section{Stopping criteria}

$$
\left\|\mathbf{u}-\mathbf{u}^{(k)}\right\|_{\mathbf{M}}^{2}=\left\|\mathbf{e}^{(k)}\right\|_{\mathbf{M}}^{2}=\sum_{j=k+1}^{n} \zeta_{j}^{2}=\left\|\mathbf{z}-\left[\begin{array}{c}
\mathbf{z}_{k} \\
0
\end{array}\right]\right\|_{2}^{2}
$$




\section{Stopping criteria}

$$
\begin{gathered}
\left\|\mathbf{u}-\mathbf{u}^{(k)}\right\|_{\mathbf{M}}^{2}=\left\|\mathbf{e}^{(k)}\right\|_{\mathbf{M}}^{2}=\sum_{j=k+1}^{n} \zeta_{j}^{2}=\left\|\mathbf{z}-\left[\begin{array}{c}
\mathbf{z}_{k} \\
0
\end{array}\right]\right\|_{2}^{2} . \\
\left\|\mathbf{A}^{T} \mathbf{u}^{(k)}-\mathbf{b}\right\|_{\mathbf{N}^{-1}}=\left|\beta_{k+1} \zeta_{k}\right| \leq \sigma_{1}\left|\zeta_{k}\right|=\|\tilde{\mathbf{A}}\|_{2}\left|\zeta_{k}\right| .
\end{gathered}
$$




\section{Stopping criteria}

$$
\begin{gathered}
\left\|\mathbf{u}-\mathbf{u}^{(k)}\right\|_{\mathbf{M}}^{2}=\left\|\mathbf{e}^{(k)}\right\|_{\mathbf{M}}^{2}=\sum_{j=k+1}^{n} \zeta_{j}^{2}=\left\|\mathbf{z}-\left[\begin{array}{c}
\mathbf{z}_{k} \\
0
\end{array}\right]\right\|_{2}^{2} . \\
\left\|\mathbf{A}^{T} \mathbf{u}^{(k)}-\mathbf{b}\right\|_{\mathbf{N}^{-1}}=\left|\beta_{k+1} \zeta_{k}\right| \leq \sigma_{1}\left|\zeta_{k}\right|=\|\tilde{\mathbf{A}}\|_{2}\left|\zeta_{k}\right| \\
\left\|\mathbf{p}-\mathbf{p}^{(k)}\right\|_{\mathbf{N}}=\left\|\mathbf{Q B}^{-1}\left(\mathbf{z}-\left[\begin{array}{c}
\mathbf{z}_{k} \\
0
\end{array}\right]\right)\right\|_{\mathbf{N}} \leq \frac{\left\|\mathbf{e}^{(k)}\right\|_{\mathbf{M}}}{\sigma_{n}}
\end{gathered}
$$




\section{Error bound}

Lower bound We can estimate $\left\|\mathbf{e}^{(k)}\right\|_{M}^{2}$ by the lower bound

$$
\xi_{k, d}^{2}=\sum_{j=k+1}^{k+d+1} \zeta_{j}^{2}<\left\|\mathbf{e}^{(k)}\right\|_{M}^{2} .
$$

Given a threshold $\tau<1$ and an integer $d$, we can stop the iterations when

$$
\xi_{k, d}^{2} \leq \tau \sum_{j=1}^{k+d+1} \zeta_{j}^{2}<\tau\|\mathbf{u}\|_{\mathbf{M}}^{2} .
$$




\section{Error bound}

Lower bound We can estimate $\left\|\mathbf{e}^{(k)}\right\|_{M}^{2}$ by the lower bound

$$
\xi_{k, d}^{2}=\sum_{j=k+1}^{k+d+1} \zeta_{j}^{2}<\left\|\mathbf{e}^{(k)}\right\|_{M}^{2} .
$$

Given a threshold $\tau<1$ and an integer $d$, we can stop the iterations when

$$
\xi_{k, d}^{2} \leq \tau \sum_{j=1}^{k+d+1} \zeta_{j}^{2}<\tau\|\mathbf{u}\|_{\mathbf{M}}^{2} .
$$

Upper bound Despite being very inexpensive, the previous estimator is still a lower bound of the error. We can use an approach inspired by the Gauss-Radau quadrature algorithm and similar to the one described in Golub-Meurant (2010). 


\section{Two examples}

\section{Stokes}

The Stokes problems have been generated using the software provided by ifiss3.0 package (Elman, Ramage, and Silvester). We use the default geometry of "Step case" and the Q2-Q1 approximation described in ifiss3.0 manual and in Elman, Silvester, and Wathen (2005).

\begin{tabular}{c|c|c|c|c}
\hline name & $\mathrm{m}$ & $\mathrm{n}$ & $\mathrm{nnz}(\mathbf{M})$ & $\mathrm{nnz}(\mathbf{A})$ \\
\hline Step1 & 418 & 61 & 2126 & 1603 \\
Step2 & 1538 & 209 & 10190 & 7140 \\
Step3 & 5890 & 769 & 44236 & 30483 \\
Step4 & 23042 & 2945 & 184158 & 126799 \\
Step5 & 91138 & 11521 & 751256 & 518897 \\
\hline
\end{tabular}

$(\mathrm{nnz}(\mathbf{M})$ is only for the symmetric part) 


\section{Two examples}

\begin{tabular}{c|c|c|c|c|c}
\hline name & \# Iter.s & $\left\|\mathbf{e}^{(k)}\right\|_{2}$ & $\left\|\mathbf{A}^{T} \mathbf{u}^{(k)}-\mathbf{b}\right\|_{2}$ & $\left\|\mathbf{p}-\mathbf{p}^{(k)}\right\|_{2}$ & $\kappa(\mathbf{B})$ \\
\hline Step1 & 30 & $6.8 \mathrm{e}-16$ & $5.1 \mathrm{e}-16$ & $1.1 \mathrm{e}-13$ & 7.6 \\
Step2 & 32 & $5.4 \mathrm{e}-14$ & $5.4 \mathrm{e}-14$ & $5.0 \mathrm{e}-12$ & 7.7 \\
Step3 & 34 & $3.8 \mathrm{e}-14$ & $2.7 \mathrm{e}-14$ & $1.0 \mathrm{e}-11$ & 7.8 \\
Step4 & 34 & $5.0 \mathrm{e}-13$ & $1.3 \mathrm{e}-13$ & $1.4 \mathrm{e}-10$ & 7.8 \\
Step5 & 35 & $1.8 \mathrm{e}-13$ & $3.1 \mathrm{e}-14$ & $1.7 \mathrm{e}-10$ & 7.8 \\
\hline
\end{tabular}

Stokes (Step) problems results $\left(d=5, \tau=10^{-8}\right)$. 


\section{Two examples}

Poisson with mixed b.c. Problems The Poisson problem is casted in its dual form as a Darcy's problem:

$$
\left\{\begin{array}{l}
\text { Find } \quad w \in \mathcal{H}=\left\{\vec{q} \mid \vec{q} \in H_{\text {div }}(\Omega), \vec{q} \cdot \mathbf{n}=0 \text { on } \partial_{N}(\Omega)\right\}, u \in L^{2}(\Omega) \\
\int_{\Omega} \vec{w} \cdot \vec{q}+\int_{\Omega} \operatorname{div}(\vec{q}) u=\int_{\partial_{D}(\Omega)} u_{D} \vec{q} \cdot \mathbf{n} \forall \vec{q} \in \mathcal{H} \\
\int_{\Omega} \operatorname{div}(\vec{w}) v=\int_{\Omega} f v \forall v \in L^{2}(\Omega) .
\end{array}\right.
$$

We approximated the spaces $\mathcal{H}$ and $L^{2}(\Omega)$ by RT0 and by piecewise constant functions respectively The matrix $\mathbf{N}$ is the mass matrix for the piecewise constant functions and it is a diagonal matrix with diagonal entries equal to the area of the corresponding triangle. The matrix $\mathbf{M}$ has been chosen such that each approximation $\mathcal{H}_{h}$ of $\mathcal{H}$ is

$$
\mathcal{H}_{h}=\left\{\mathbf{q} \in \mathbf{R}^{m}\|\mathbf{q}\|_{\mathcal{H}_{h}}^{2}=\mathbf{q}^{T} \mathbf{M q}\right\} .
$$

Therefore, denoting by $\mathbf{W}$ the mass matrix for $\mathcal{H}_{h}$, we have

$$
\mathbf{M}=\mathbf{W}+\mathbf{A N}^{-1} \mathbf{A}^{\top}
$$




\section{Two examples}

Poisson with mixed b.c. Problems

\begin{tabular}{c|c|c|c|c}
\hline$h=2^{-k}$ & $\mathrm{~m}$ & $\mathrm{n}$ & $\mathrm{nnz}(\mathbf{M})$ & $\mathrm{nnz}(\mathbf{A})$ \\
\hline $2^{-6}$ & 12288 & 8192 & 36608 & 24448 \\
$2^{-7}$ & 49152 & 32768 & 146944 & 98048 \\
$2^{-8}$ & 196608 & 131072 & 588800 & 392704 \\
$2^{-9}$ & 786432 & 524288 & 2357248 & 1571840 \\
\hline \multicolumn{5}{c}{$(\mathrm{nnz}(\mathbf{M})$ is only for the symmetric part) }
\end{tabular}

With the chosen boundary conditions, it is easy to verify that the continuous solution $u$ is $u(x, y)=x$.

We point out that the pattern of $\mathbf{W}$ is structurally equal to the pattern $\mathbf{A} \mathbf{N}^{-1} \mathbf{A}^{T}$. 


\section{Two examples}

\begin{tabular}{c|c|c|c|c|c}
\hline name & $\#$ Iter.s & $\left\|\mathbf{e}^{(k)}\right\|_{2}$ & $\left\|\mathbf{A}^{T} \mathbf{u}^{(k)}-\mathbf{b}\right\|_{2}$ & $\left\|\mathbf{p}-\mathbf{p}^{(k)}\right\|_{2}$ & $\kappa(\mathbf{B})$ \\
\hline$h=2^{-6}$ & 10 & $2.8 \mathrm{e}-12$ & $2.9 \mathrm{e}-16$ & $4.1 \mathrm{e}-11$ & 1.05 \\
$h=2^{-7}$ & 10 & $9.7 \mathrm{e}-12$ & $3.0 \mathrm{e}-16$ & $2.6 \mathrm{e}-10$ & 1.05 \\
$h=2^{-8}$ & 10 & $2.5 \mathrm{e}-11$ & $3.0 \mathrm{e}-16$ & $7.9 \mathrm{e}-10$ & 1.05 \\
$h=2^{-9}$ & 10 & $2.9 \mathrm{e}-10$ & $2.8 \mathrm{e}-16$ & $1.3 \mathrm{e}-08$ & 1.05 \\
\hline
\end{tabular}

Poisson with mixed b.c. data and RT0 problem results $(d=5$,

$$
\tau=10^{-8} \text { ). }
$$




\section{Symmetric Quasi-Definite Systems}

$\left[\begin{array}{rr}\mathbf{M} & \mathbf{A} \\ \mathbf{A}^{T} & -\mathbf{N}\end{array}\right]\left[\begin{array}{l}\mathbf{x} \\ \mathbf{y}\end{array}\right]=\left[\begin{array}{l}\mathbf{f} \\ \mathbf{g}\end{array}\right] \quad$ where $\quad \mathbf{M}=\mathbf{M}^{T} \succ 0, \mathbf{N}=\mathbf{N}^{T} \succ 0$.

- Interior-point methods for LP, QP, NLP, SOCP, SDP, ...

- Regularized/stabilized PDE problems

- Regularized least squares

- How to best take advantage of the structure? 


\section{Main Property}

Theorem (Vanderbei, 1995)

If $\mathbf{K}$ is $S Q D$, it is strongly factorizable, i.e., for any permutation matrix $\mathbf{P}$, there exists a unit lower triangular $\mathbf{L}$ and a diagonal $\mathbf{D}$ such that $\mathbf{P}^{T} \mathbf{K P}=\mathbf{L D L}^{T}$.

- Cholesky-factorizable

- Used to speed up factorization in regularized least-squares (Saunders) and interior-point methods (Friedlander and O.)

- Stability analysis by Gill, Saunders, Shinnerl (1996). 


\section{Iterative Methods I}

Facts: SQD systems are symmetric, non-singular, square and indefinite. 


\section{Iterative Methods I}

Facts: SQD systems are symmetric, non-singular, square and indefinite.

- MINRES

- SYMMLQ

- (F)GMRES??

QMRS???? 


\section{Iterative Methods I}

Facts: SQD systems are symmetric, non-singular, square and indefinite.

- MINRES

- SYMMLQ

- (F)GMRES??

QMRS????

Fact: ... none exploits the SQD structure. 


\section{Iterative Methods I}

Facts: SQD systems are symmetric, non-singular, square and indefinite.

- MINRES

- SYMMLQ

- (F)GMRES??

QMRS????

Fact: ... none exploits the SQD structure.

If the system were definite, we would like to use CG. 


\section{Related Problems: an example}

$$
\left[\begin{array}{lr}
\mathbf{M} & \mathbf{A} \\
\mathbf{A}^{T} & -\mathbf{N}
\end{array}\right]\left[\begin{array}{l}
\mathbf{x} \\
\mathbf{y}
\end{array}\right]=\left[\begin{array}{l}
\mathbf{b} \\
0
\end{array}\right]
$$




\section{Related Problems: an example}

$$
\left[\begin{array}{lr}
\mathbf{M} & \mathbf{A} \\
\mathbf{A}^{T} & -\mathbf{N}
\end{array}\right]\left[\begin{array}{l}
\mathbf{x} \\
\mathbf{y}
\end{array}\right]=\left[\begin{array}{l}
\mathbf{b} \\
0
\end{array}\right]
$$

are the optimality conditions of

$\min _{\mathbf{y} \in \mathbf{R}^{m}} \frac{1}{2}\left\|\left[\begin{array}{c}\mathbf{A} \\ \mathbf{I}\end{array}\right] \mathbf{y}-\left[\begin{array}{c}\mathbf{b} \\ 0\end{array}\right]\right\|_{E_{+}^{-1}}^{2} \equiv \min _{y \in \mathbf{R}^{m}} \frac{1}{2}\left\|\left[\begin{array}{cc}\mathbf{M}^{-\frac{1}{2}} & 0 \\ 0 & \mathbf{N}^{\frac{1}{2}}\end{array}\right]\left(\left[\begin{array}{c}\mathbf{A} \\ \mathbf{I}\end{array}\right] \mathbf{y}-\left[\begin{array}{l}\mathbf{b} \\ 0\end{array}\right]\right)\right\|_{2}^{2}$ 


\section{Generalized Least Squares}

Normal equations: $\left(\mathbf{A}^{T} \mathbf{M}^{-1} \mathbf{A}+\mathbf{N}\right) \mathbf{y}=\mathbf{A}^{T} \mathbf{M}^{-1} \mathbf{b}$. 


\section{Generalized Least Squares}

Normal equations: $\left(\mathbf{A}^{T} \mathbf{M}^{-1} \mathbf{A}+\mathbf{N}\right) \mathbf{y}=\mathbf{A}^{T} \mathbf{M}^{-1} \mathbf{b}$.

At $k$-th iteration, seek $y \approx \mathbf{y}_{k}:=\mathbf{V}_{k} \overline{\mathbf{y}}_{k}$ :

$$
\left(\tilde{\mathbf{B}}_{k}^{T} \tilde{\mathbf{B}}_{k}+\mathbf{I}\right) \overline{\mathbf{y}}_{k}=\tilde{\mathbf{B}}_{k}^{T} \beta_{1} \mathbf{e}_{1}
$$




\section{Generalized Least Squares}

Normal equations: $\left(\mathbf{A}^{T} \mathbf{M}^{-1} \mathbf{A}+\mathbf{N}\right) \mathbf{y}=\mathbf{A}^{T} \mathbf{M}^{-1} \mathbf{b}$.

At $k$-th iteration, seek $y \approx \mathbf{y}_{k}:=\mathbf{V}_{k} \overline{\mathbf{y}}_{k}$ :

$$
\left(\tilde{\mathbf{B}}_{k}^{T} \tilde{\mathbf{B}}_{k}+\mathbf{I}\right) \overline{\mathbf{y}}_{k}=\tilde{\mathbf{B}}_{k}^{T} \beta_{1} \mathbf{e}_{1}
$$

i.e.:

$$
\min _{\overline{\mathbf{y}} \in \mathbf{R}^{k}} \frac{1}{2}\left\|\left[\begin{array}{c}
\tilde{\mathbf{B}}_{k} \\
\mathbf{l}
\end{array}\right] \overline{\mathbf{y}}-\left[\begin{array}{c}
\beta_{1} \mathbf{e}_{1} \\
0
\end{array}\right]\right\|_{2}^{2}
$$




\section{Generalized Least Squares}

Normal equations: $\left(\mathbf{A}^{T} \mathbf{M}^{-1} \mathbf{A}+\mathbf{N}\right) \mathbf{y}=\mathbf{A}^{T} \mathbf{M}^{-1} \mathbf{b}$.

At $k$-th iteration, seek $y \approx \mathbf{y}_{k}:=\mathbf{V}_{k} \overline{\mathbf{y}}_{k}$ :

$$
\left(\tilde{\mathbf{B}}_{k}^{T} \tilde{\mathbf{B}}_{k}+\mathbf{I}\right) \overline{\mathbf{y}}_{k}=\tilde{\mathbf{B}}_{k}^{T} \beta_{1} \mathbf{e}_{1}
$$

i.e.:

$$
\min _{\overline{\mathbf{y}} \in \mathbf{R}^{k}} \frac{1}{2}\left\|\left[\begin{array}{c}
\tilde{\mathbf{B}}_{k} \\
\mathbf{l}
\end{array}\right] \overline{\mathbf{y}}-\left[\begin{array}{c}
\beta_{1} \mathbf{e}_{1} \\
0
\end{array}\right]\right\|_{2}^{2}
$$

or:

$$
\left[\begin{array}{cc}
\mathbf{I} & \tilde{\mathbf{B}}_{k} \\
\tilde{\mathbf{B}}_{k}^{T} & -\mathbf{I}
\end{array}\right]\left[\begin{array}{c}
\overline{\mathbf{x}}_{k} \\
\overline{\mathbf{y}}_{k}
\end{array}\right]=\left[\begin{array}{c}
\beta_{1} \mathbf{e}_{1} \\
0
\end{array}\right]
$$




\section{Generalized LSQR}

Solve

$$
\min _{\overline{\mathbf{y}} \in \mathbf{R}^{k}} \frac{1}{2}\left\|\left[\begin{array}{c}
\tilde{\mathbf{B}}_{k} \\
\mathbf{l}
\end{array}\right] \overline{\mathbf{y}}-\left[\begin{array}{c}
\beta_{1} \mathbf{e}_{1} \\
0
\end{array}\right]\right\|_{2}^{2}
$$

by specialized Givens Rotations (Eliminate $\mathbf{I}$ first and $\mathbf{R}_{k}$ will be upper bidiagonal) 


\section{Generalized LSQR}

Solve

$$
\min _{\overline{\mathbf{y}} \in \mathbf{R}^{k}} \frac{1}{2}\left\|\left[\begin{array}{c}
\tilde{\mathbf{B}}_{k} \\
\mathbf{l}
\end{array}\right] \overline{\mathbf{y}}-\left[\begin{array}{c}
\beta_{1} \mathbf{e}_{1} \\
0
\end{array}\right]\right\|_{2}^{2}
$$

by specialized Givens Rotations (Eliminate $\mathbf{I}$ first and $\mathbf{R}_{k}$ will be upper bidiagonal)

$$
\min _{\overline{\mathbf{y}} \in \mathbf{R}^{k}} \frac{1}{2}\left\|\left[\begin{array}{c}
\tilde{\mathbf{R}}_{k} \\
0
\end{array}\right] \overline{\mathbf{y}}-\left[\begin{array}{c}
\phi_{k} \\
0
\end{array}\right]\right\|_{2}^{2} .
$$




\section{Generalized LSQR}

Solve

$$
\min _{\overline{\mathbf{y}} \in \mathbf{R}^{k}} \frac{1}{2}\left\|\left[\begin{array}{c}
\tilde{\mathbf{B}}_{k} \\
\mathbf{l}
\end{array}\right] \overline{\mathbf{y}}-\left[\begin{array}{c}
\beta_{1} \mathbf{e}_{1} \\
0
\end{array}\right]\right\|_{2}^{2}
$$

by specialized Givens Rotations (Eliminate $\mathbf{I}$ first and $\mathbf{R}_{k}$ will be upper bidiagonal)

$$
\min _{\overline{\mathbf{y}} \in \mathbf{R}^{k}} \frac{1}{2}\left\|\left[\begin{array}{c}
\tilde{\mathbf{R}}_{k} \\
0
\end{array}\right] \overline{\mathbf{y}}-\left[\begin{array}{c}
\phi_{k} \\
0
\end{array}\right]\right\|_{2}^{2} .
$$

As in Paige-Saunders '82 we can build recursive expressions of $\mathbf{y}_{k}$

$$
\mathbf{y}_{k+1}=\mathbf{y}_{k}+\mathbf{d}_{k} \phi_{k} \quad\left(\mathbf{D}_{k}=\mathbf{V}_{k} \mathbf{R}_{k}^{-1}\right)
$$

and we have that

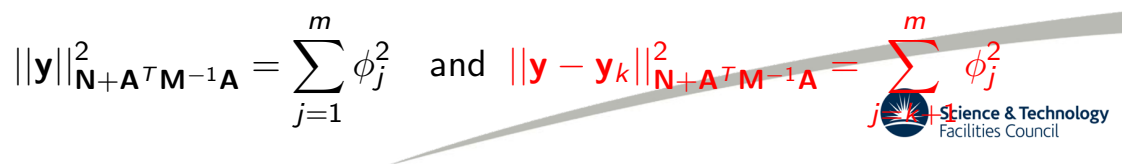




\section{Conclusions}

- Nice relation between the algebraic error and the approximation error for mixed finite-element method (See A. RAL-TR-2010-008) 


\section{Conclusions}

- Nice relation between the algebraic error and the approximation error for mixed finite-element method (See A. RAL-TR-2010-008)

- Dominique Orban and I are analysing several other variants

- Craig,

- GLSMR

and the numerical results validate the theory. 


\section{Conclusions}

- Nice relation between the algebraic error and the approximation error for mixed finite-element method (See A. RAL-TR-2010-008)

- Dominique Orban and I are analysing several other variants

- Craig,

- GLSMR

and the numerical results validate the theory.

- A. and Orban "Iterative methods for symmetric quasi definite systems" in preparation. WORK IN PROGRESS 